\title{
A aplicação do Storytelling nas pesquisas em Turismo: Uma Revisão Bibliométrica, Sistemática e Integrativa da Literatura
}

\author{
The application of Storytelling in Tourism research: A \\ Bibliometric, Systematic and Integrative Review of \\ Literature
}

Franciele Cristina Manosso

Professora Substituta do Departamento de Turismo (DETUR) da Universidade Federal do Paraná - UFPR, Curitiba/PR, Brasil

E-mail: francimanosso@gmail.com

Thays Cristina Domareski Ruiz

Professora adjunta do Curso de Turismo da Universidade Federal do Paraná - UFPR, Curitiba/PR, Brasil

E-mail: thaysdomareski@gmail.com

Marcia Shizue Massukado Nakatani

Professora adjunta do Curso de Turismo e professora permanente do Programa de PósGraduação em Turismo (PPGTUR) da Universidade Federal do Paraná - UFPR, Curitiba/PR, Brasil

E-mail: marcia.nakatani@gmail.com

Artigo recebido em: 16-01-2019

Artigo aprovado em: 28-04-2020 


\section{RESUMO}

O storytelling tem o poder de informar, persuadir, emocionar, promover apoio e iniciativas na sociedade. Com a mudança no comportamento do consumidor, a popularização da economia da experiência e as inovações tecnológicas, o uso do storytelling aumentou nos últimos anos. Pois, este, enquanto ferramenta vem sendo trabalhado como meio de promover negócios, produtos e serviços e, no caso do turismo, destinos. Com base em um estudo exploratório, este artigo realizou revisões bibliométrica, sistemática e integrativa da literatura para traçar um panorama a respeito do uso do storytelling nas pesquisas acadêmicas em turismo. Para tal, foram empregados os descritores storytelling, marketing e tourism nas bases de dados: Taylor and Francis, Science Direct, Portal da Capes e Scopus. Com as buscas foi possível compilar um corpus de 22 artigos. Os indicadores bibliométricos apontaram que o estudo da temática é recente, visto que os artigos foram publicados nos últimos doze anos e que há uma dispersão espacial dos pesquisadores que investigam a temática. A revisão sistemática apontou dois objetos de estudo: a experiência turística e o marketing de destinos. Portanto, pode-se concluir que a abordagem do storytelling tem sido empregada como ferramenta de marketing tanto em organizações quanto nos destinos, utilizando-o como estratégia de comunicação da marca, evidenciando sua característica de propagar a partir de narrativas, as experiências vivenciadas e transformar as principais características do destino em histórias.

Palavras-Chave: Turismo. Storytelling. Marketing. Pesquisa. Revisão de Literatura.

\section{ABSTRACT}

Storytelling has the power to inform, persuade, thrill, promote support and initiatives in society. With the changes in consumer behaviour, the popularisation of the economy of experience and technological innovations, the use of storytelling has increased in recent years. It has been used as tool to promote business, products and services and, in the case of tourism, destinations. Based on an exploratory study, this paper carried out bibliometric, systematic and integrative literature reviews to outline the use of storytelling in academic research in tourism. To that end, the descriptors were used: storytelling, marketing and tourism in the databases: Taylor and Francis, Science Direct, Portal de Capes and Scopus. With the searches, it was possible to compile a corpus of 22 articles. The bibliometric indicators indicated that the study of the thematic is recent since the articles were published in the last twelve years and that there is a spatial dispersion of the researchers that investigate the subject. The systematic review pointed to two objects of study: tourism experience and destination marketing. Therefore, it can be concluded that the storytelling approach has been used as a marketing tool both in organisations and in destinations, using it as a communication strategy of the brand, evidencing its characteristic of propagating from narratives, experiences and transform the main attributes of destiny in stories.

Keywords: Tourism. Storytelling. Marketing. Research. Literature Review. 


\section{INTRODUÇÃO}

A evolução da tecnologia e a inovação, impulsionadas pelo processo de globalização, permitiram evidenciar a importância da revolução da internet e dos meios de comunicação online no processo de transformação do setor turístico. A internet revela-se um instrumento abrangente, principalmente pela possibilidade de interatividade dos consumidores entre si e para/com suas marcas e empresas, além de oferecer acesso a milhares de informações (Alves, Costa, \& Perinotto, 2017).

Os gestores de marketing enfrentam no cenário da comunicação constantes mudanças, tais quais: o surgimento de uma nova ferramenta e/ou tecnologia, a adoção em massa de canais de mídias sociais e, principalmente o protagonismo de outras vozes (e.g. user generated content) no contexto do marketing de destinos. Neste sentido e corroborando com Bryon (2012) o marketing considera o desenvolvimento de mensagens, destaca a ideia de narrativas ou histórias contadas pelos turistas e para os turistas que retratem experiências no destino, ou seja, o uso do conceito de storytelling para o marketing turístico.

O storytelling é a maneira mais natural de comunicação desde o início da história do homem (Patterson \& Brown, 2005). Pois, pode ser considerado uma ferramenta de percepção e um dispositivo para a expressão emocional em relação ao mundo, contemplando como as pessoas o vêem (Kim \& Jeong, 2010). Sole e Wilson (1999 ${ }^{1}$ citado por Lee \& Shin, 2015, p. 285) afirmam que o storytelling "é definido como compartilhar conhecimento ou experiência através de uma história para compreensão de uma ideia complicada, conceito e relação casual".

Em essência, o storytelling é uma forma de narrativa, isto é, de contar as ocorrências cotidianas (Fog, Budtz, \& Yakaboylu, 2005). Ou seja, deve ser entendido como a arte interativa de usar palavras e ações, e seus propósitos revelam os elementos de uma história e estimulam a imaginação do ouvinte (National Storytelling Network, 2011). Consequentemente, a narrativa surge dos esforços interativos, cooperativos e coordenados de duas partes envolvidas: os contadores de histórias e o público, e é, portanto, uma comunicação de duas vias em que as respostas dos ouvintes desempenham um papel ativo (Howison, Higgins-Desbiolles, \& Sun, 2017).

O storytelling pode ser visto como o conceito que combina a articulação de entendimentos que define a comunicação e uma narrativa envolvente que forma a história. Pode ser considerado um modo direto de abordar a participação e a interação dos visitantes (Gabriel

\footnotetext{
${ }^{1}$ Sole D., \& Wilson D. (1999). Storytelling in organizations: the power and traps of using stories to share knowledge in organizations. Train Dev, 53, 44-52.
} 
2000; Nielsen, 2017), bem como, ser debatido como uma estratégia eficaz de manter os clientes existentes e atrair os em potencial (Jensen, 1999; Delgadillo \& Esealas, 2004); se tornar um novo modelo de negócios (Montoro-Sanchez, 2009; Lee \& Shin, 2015), ou ainda ser utilizado pelas organizações e empresas para explicar políticas ou missões e efetuar mudanças (Boje, 1995; O'Gorman \& Gillespie, 2010). Cabe destacar que a intenção da narrativa não é estabelecer um final, sendo que a história pode continuar dependendo do narrador e do público, que criam significados a partir das palavras e chegam a um melhor entendimento de seu lugar no processo de comunicação (Garrett, 1996; Powell, Weems, \& Owle, 2007; Price, Kallam, \& Love, 2009).

Acompanhando o desenvolvimento digital, o storytelling também está sendo utilizado nesse ambiente. O modelo clássico de narração digital foi desenvolvido pelo The Center for Digital Storytelling nos anos 1990. Lambert (2006) afirma que o início da narrativa digital data de 1993, destacando Dana Atchey, John Lambert, Nina Mullen e Patrick Milligan.

O storytelling digital é uma nova forma de narrativa que compartilha características de meios mais tradicionais de contar histórias com novos tipos de narrativas de auto-expressão, assim pode ser uma forma de auto-apresentação através de múltiplos meios (Hull \& Nelson, 2009; Alcantud-Díaz, Vayá, \& Gregori-Signes, 2014). Alguns autores conhecidos no campo da narrativa digital, como Robin (2006, 2008a, 2008b) e Barrett (2006a, 2006b) afirmam que esta é uma boa maneira de envolver as pessoas em formas tradicionais e inovadoras de contar uma história (Alcantud-Díaz, Vayá, \& Gregori-Signes, 2014).

Especificamente no contexto do turismo, o storytelling recebeu maior atenção dos pesquisadores como um meio eficaz de comunicar e, deveras, proporcionar experiências turísticas (McCabe \& Foster, 2006; Chronis, 2008; Youssef, Leicht, \& Marongiu, 2018). Na área turística, o storytelling vem sendo trabalhado como uma abordagem integrada ao marketing de destinos (Drew \& Woodside, 2011; Chronis, 2012; Lund, Cohen, \& Scarles, 2017; Youssef, Leicht, \& Marongiu, 2018), visto que no turismo, concentra seus esforços no comportamento das viagens e segue uma estrutura que beneficia a prática de gestão turística. As pesquisas que correlacionam turismo, marketing e storytelling focam os protagonistas que interpretam jornadas, desdobramentos dramáticos de eventos e explicações arquetípicas. Esse foco fornece credibilidade para o marketing de um destino usando a narrativa sobre um protagonista em uma jornada versus discursos propagandísticos sobre os benefícios derivados das características de um local (Adaval \& Wyer, 1998; Woodside \& Megehee, 2009).

Em tal conjuntura e considerando que a produção de sentidos está presente no turismo, quer na experiência vivenciada pelo turista ou iminente à ideia de intangibilidade do destino e do produto turístico, este estudo tem como objetivo analisar a aplicação do storytelling nos 
estudos em turismo mediante pesquisa nas principais bases de dados de publicações científicas nacionais e internacionais. Com isso espera-se, a partir dessa discussão, ampliar a compreensão do turismo enquanto fenômeno comunicacional (Wainberg, 2003) e trazer contribuições de como a produção e narração de histórias, independente do interlocutor, pode ser um instrumento de promoção das localidades como destinos e produtos turísticos (Nakatani, Gomes, \& Nunes, 2017). Visto a aplicação das revisões bibliométrica, sistemática e integrativa da literatura, este trabalho apresenta na sequência a metodologia empregada. O tópico posterior debate os resultados e a discussão a partir da investigação realizada, finalizando com as conclusões do estudo.

\section{PROCEDIMENTOS METODOLÓGICOS}

O storytelling enquanto ferramenta vem sendo trabalhado nas mais diversas áreas de atuação, principalmente, como um meio de promover negócios, produtos e serviços e, no caso do turismo, destinos (Hsiao, Lu, \& Lian, 2013). Para o desenvolvimento teórico e metodológico do presente trabalho serão empregadas como ferramentas: a revisão bibliométrica; sistemática e integrativa da literatura para que se possa traçar um panorama a respeito do uso do storytelling nas pesquisas acadêmicas em turismo.

A revisão bibliométrica se caracteriza como um método estatístico que permite o mapeamento e a geração de diferentes indicadores de tratamento (Guedes \& Borschiver, 2005). Macedo, Botelho e Duarte (2010) salientam que tal ferramenta de pesquisa permite encontrar uma quantidade restrita de periódicos que comportam os artigos mais relevantes publicados acerca de determinada temática. À tal perspectiva, Souza (2013) acrescenta que a revisão bibliométrica pode ser considerada uma maneira de medir a literatura dos documentos e outros meios de comunicação permitindo a identificação de tendências e crescimentos, usuários e autores, a verificação da cobertura das revistas, bem como torna possível mensurar a disseminação da informação a respeito da temática estudada.

Para a concretização da presente pesquisa a revisão bibliométrica foi realizada nas seguintes bases de dados: Portal Capes; Science Direct; Scopus e Taylor and Francis, sendo realizada em dois momentos. A etapa preliminar é apresentada na Figura 1. 


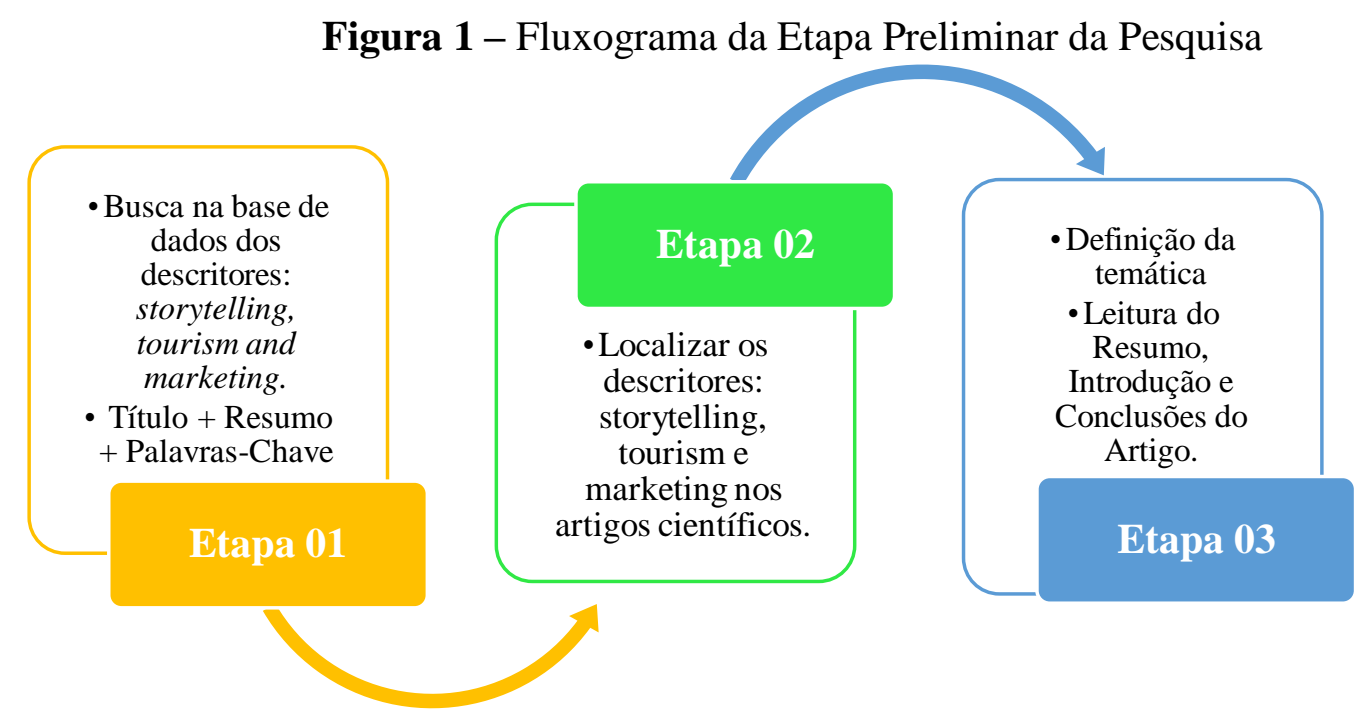

Fonte: Elaborado pelas autoras, 2019.

Como resultados da pesquisa preliminar foram recuperados 3028 artigos nas quatro bases analisadas e, após filtragem manual foram retirados os repetidos e aqueles que não eram correlatos ao tema, desta forma, chegou-se a 63 artigos conforme o que se destaca na Tabela 1.

\begin{tabular}{|ccc|} 
& Tabela 1 - Número de Artigos - Busca Preliminar \\
Base de Dados & Artigos Etapa 01 & Artigos Etapa 02 \\
\hline Portal da Capes & 1006 & 19 \\
\hline Science Direct & 257 & 10 \\
\hline Scopus & 20 & 15 \\
\hline Taylor and Francis & 1745 & 19 \\
\hline & 3028 & 63 \\
\hline
\end{tabular}

Fonte: Elaborado pelas autoras, 2019.

A segunda etapa da pesquisa bibliométrica foi realizada nas mesmas bases de dados, contudo considerando a busca avançada. Como cada base tem um sistema único de recuperação de publicações, as buscas foram realizadas conforme descrito na Tabela 2.

\begin{tabular}{|c|c|c|c|c|c|}
\hline Base De Dados & Descritores & Boleanos & Campo & Filtro & Artigos \\
\hline Portal da Capes & tourism, storytelling & e & assunto & nenhum & 12 \\
\hline Science Direct & tourism, storytelling & $\mathrm{e}$ & $\begin{array}{l}\text { título e resumo e } \\
\text { palavras-chaves }\end{array}$ & nenhum & 10 \\
\hline Scopus & $\begin{array}{c}\text { tourism, marketing, } \\
\text { storytelling }\end{array}$ & $\mathrm{e} ; \mathrm{ou}$ & $\begin{array}{l}\text { título ou resumo ou } \\
\text { palavras-chaves }\end{array}$ & nenhum & 20 \\
\hline \multirow[t]{2}{*}{$\begin{array}{c}\text { Taylor and } \\
\text { Francis }\end{array}$} & storytelling & $\mathrm{e}$ & $\begin{array}{l}\text { título e palavras- } \\
\text { chaves }\end{array}$ & $\begin{array}{c}\text { Área: Tourism, } \\
\text { Hospitality and } \\
\text { Events }\end{array}$ & 8 \\
\hline & & & & & 50 \\
\hline
\end{tabular}

Fonte: Elaborado pelas autoras, 2019. 
Dos 50 artigos recuperados na busca avançada, 8 se repetiam em mais de uma base. Assim, o número final de artigos recuperados foram 42. De tal modo, os 105 artigos de ambas as buscas (preliminar e avançada) foram reunidos em uma tabela para verificar a existência de duplicação de publicações (44 artigos apareceram em ambas as buscas). Na sequência foram verificados aqueles que havia acesso ao conteúdo completo do artigo (4 foram excluídos) e então reunidos chegou-se ao total de 57 artigos que foram recuperados em ambas as buscas.

A etapa seguinte consistiu em delimitar, a partir da leitura dos 57 artigos aqueles em que os descritores tourism, storytelling e marketing apareciam de forma expressiva no conteúdo. Logo, foram retirados aqueles em que a menção a um ou mais descritores se atinham somente às referências, ou ao resumo ou às palavras-chave, chegando então ao corpus de 22 artigos para serem analisados. A Figura 2 apresenta o fluxograma das etapas realizadas para a compilação do corpus da pesquisa.

Figura 2 - Fluxograma do Corpus da Pesquisa

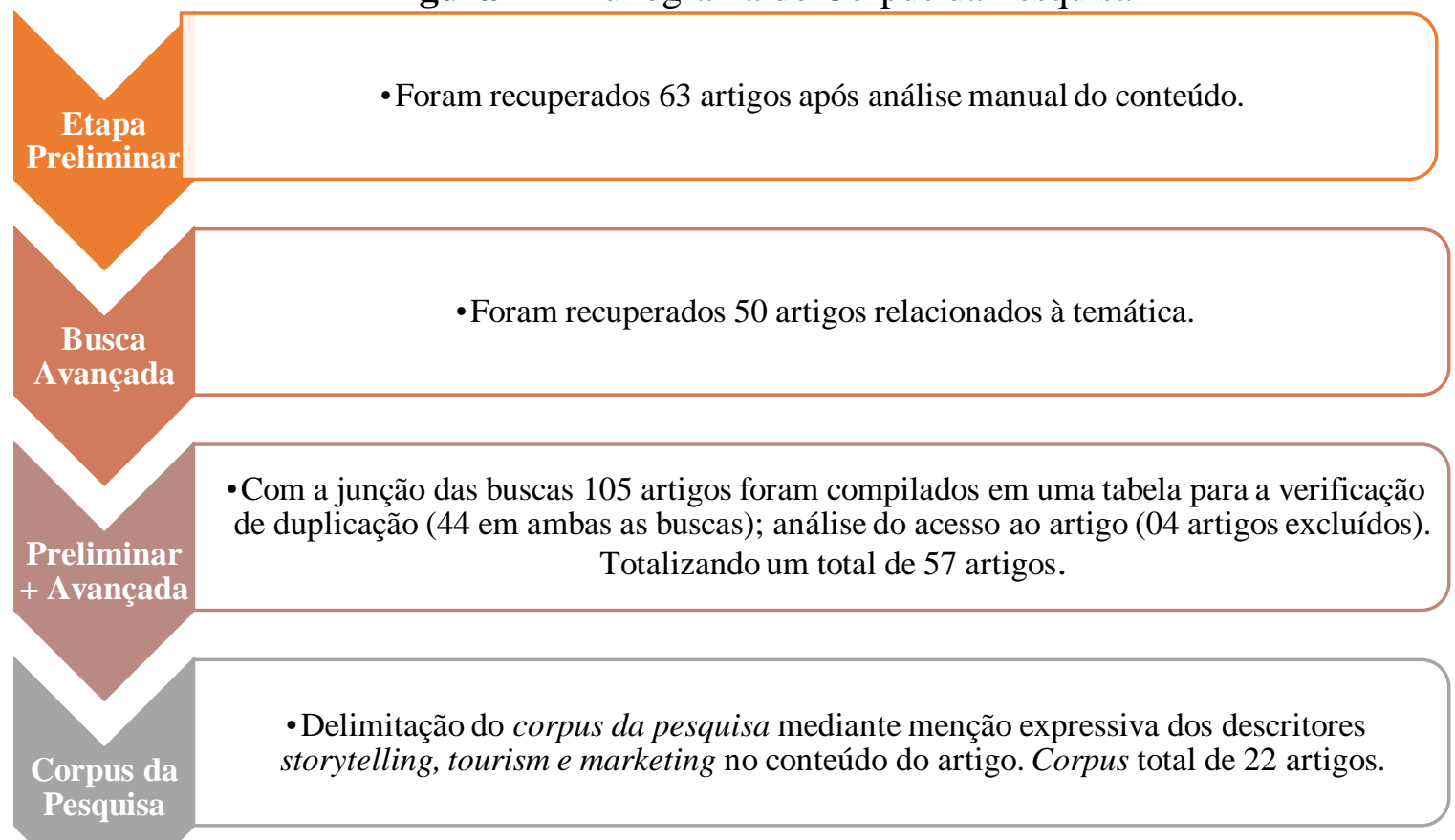

Fonte: Elaborado pelas autoras, 2019.

Em complementação à bibliometria, emprega-se a revisão sistemática que, de acordo com Lopes e Fracolli (2008), é definida como uma síntese de estudos primários que abrangem objetivos, materiais e métodos explicitados, sendo conduzida de acordo com uma metodologia clara e reprodutível. Clarke e Horton (2001) ponderam que a revisão sistemática deve ser considerada como um tipo de investigação focada na reunião, avaliação crítica e condução de 
uma síntese de resultados obtidos a partir de múltiplos estudos primários. Para uma revisão sistemática completa e abrangente alguns passos devem ser seguidos, os quais: definição do objeto da revisão; identificação da literatura e seleção dos estudos passíveis de inclusão (Moscardi, Pinto, Gomes, \& Nakatani, 2017). Assim para a revisão sistemática foi identificado e descrito: como o storytelling é utilizado no artigo, o que do turismo foi estudado e, qual a relação com o marketing.

Como recurso metodológico final foi empregada a revisão integrativa que, de acordo com Broome (2000), é realizada através da reunião, de maneira resumida, das conclusões gerais dos estudos compilados na temática. Para diferentes autores a revisão integrativa é considerada uma das revisões mais amplas (Whittemore \& Knafl, 2005), pois para a utilização desta ferramenta são necessárias a realização das seguintes etapas: formulação do problema; coleta de dados ou busca na literatura; avaliação dos dados; análise dos dados; e a interpretação e apresentação dos resultados (Russel, 2005).

Albach (2015) agrega a tal conjuntura que a revisão integrativa da literatura, juntamente aos aspectos quantitativos e qualitativos abrangidos pela revisão bibliométrica e sistemática, permite a identificação de panoramas para entender os estudos em turismo. Para compreender o uso do storytelling a partir da revisão integrativa tomou-se como base as perspectivas do destino e do turista e, assim as publicações foram apresentadas e discutidas de maneira resumida no tópico seguinte.

Em resumo, a metodologia de pesquisa do presente artigo foi trabalhada conforme a Figura 3.

Figura 3 - Fluxograma Metodológico da Pesquisa

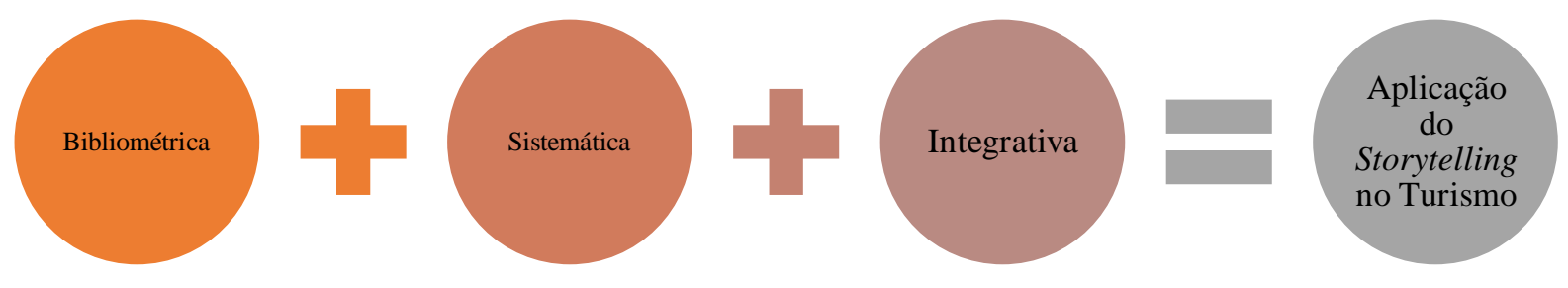

Fonte: Elaborado pelas Autoras, 2019.

O tópico a seguir aborda os resultados e a discussão oriunda da aplicação das ferramentas metodológicas apresentadas no presente capítulo. 


\section{APRESENTAÇÃO E DISCUSSÃO DOS RESULTADOS}

Histórias conectam pessoas, criam experiências compartilhadas e são essenciais para o turismo prosperar. Muitas economias dependentes do turismo desejam atrair os visitantes para seus destinos em uma concorrência séria que exige uma estratégia ponderada e informada (Howison, Higgins-Desbiolles, \& Sun, 2017).

Neste estudo, o storytelling é visto como uma abordagem experimental estratégica para o marketing turístico, onde torna-se uma bússola que direciona o desenvolvimento do destino tanto interna quanto externamente (Fog, Budtz, Munch, \& Blanchette, 2010). As histórias podem atuar como uma estrutura holística, comunicando os valores centrais da oferta. Logo, a abordagem do storytelling conecta e enquadra as experiências e, além disso, tem a capacidade de coordenar e envolver vários interessados no destino em um processo de contar histórias (Olsson, Therkelsen, \& Mossberg, 2016).

Neste sentido, os artigos analisados e apresentados a seguir abordam de alguma maneira a ferramenta de storytelling e apresentam como conceito estrutural a experiência, evidenciando seu uso tanto da perspectiva de gestão do destino quanto do consumo, ou seja, do turista.

\subsection{Indicadores bibliométricos e revisão sistemática do uso do storytelling em} estudos em turismo

Os indicadores bibliométricos utilizados para mapear o uso do storytelling nas publicações recuperadas foram: ano de publicação, periódico em que foi publicado, autoria, filiação acadêmica e país dos autores. Os 22 artigos analisados foram publicados após o ano de 2006, com destaque para 16 trabalhos disseminados nos últimos 6 anos; a média de 'idade' das publicações é o ano de 2013 e o ano em que houve maior número foi 2017 (cinco). Conclui-se a partir desses dados que as publicações que retratam o uso do storytelling nos estudos em turismo são recentes.

Outro dado é a presença de 17 periódicos diferentes que no período de quase 10 anos realizaram a publicação desses trabalhos, dos quais 3 na Journal of Travel and Tourism Marketing, três na Scandinavian Journal of Hospitality and Tourism, dois na Current Issues in Tourism e, um na revista francesa Mondes du Tourisme.

Foram localizados 44 autores diferentes nas publicações analisadas, dos quais Arch G. Woodside à época filiado ao Boston College/EUA (Carroll School of Management, Department of Marketing) escreveu 3 artigos (Martin, Woodside, \& Dehuang, 2007; Woodside, Cruickshank, \& Dehuang, 2007; Hsu, Dehuang, \& Woodside, 2009) e seu colega Ning 
Dehuang, da Kunming University of Science and Technology/China, foi co-autor em 3 destes. Registra-se, também, um total de 16 países de filiação dos autores, dos quais citam-se pesquisadores filiados a universidades (31), a empresa de consultoria (1) ou a órgão oficial de turismo (1).

\begin{tabular}{|c|c|c|}
\hline & País de filiação acadêmica & Autores \\
\hline \multirow{8}{*}{ Europa } & Reino Unido & 4 \\
\hline & Suécia & 4 \\
\hline & Turquia & 4 \\
\hline & França & 3 \\
\hline & Itália & 2 \\
\hline & Noruega & 2 \\
\hline & Bélgica & 1 \\
\hline & Dinamarca & 1 \\
\hline \multirow{2}{*}{ Oceania } & Nova Zelândia & 2 \\
\hline & Austrália & 1 \\
\hline \multirow{2}{*}{ Américas } & Estados Unidos & 7 \\
\hline & Canadá & 2 \\
\hline \multirow{5}{*}{ Ásia } & Taiwan & 7 \\
\hline & Hong Kong & 4 \\
\hline & China & 2 \\
\hline & Coreia do Sul & 1 \\
\hline & & $47^{2}$ \\
\hline
\end{tabular}

Fonte: Elaborado pelas autoras, 2019.

A distribuição espacial dos artigos analisados revela que há uma difusão de produção de conhecimento sobre a aplicação do storytelling em pesquisas em turismo, visto que foram localizadas publicações de autores filiados a instituições sediadas em países da Europa (21), Ásia (14), América do Norte (9) e Oceania (3).

A despeito da revisão sistemática destaca-se que a distribuição espacial - caracterizada pela dispersão da produção de conhecimento sobre o tema, ainda que temporalmente concomitante - também influenciou os objetos de estudo, conforme Tabela 4.

Tabela 4 - Objetos de Estudo

\begin{tabular}{|l|l|}
\hline Temática & Número de Artigos \\
\hline Turista e Experiência & 08 \\
\hline Marketing de Destinos & 07 \\
\hline Turismo Cultural & 03 \\
\hline Mercado de Cruzeiros de Luxo & 01 \\
\hline Turismo Voluntário & 01 \\
\hline Marca de Destinos Turísticos & 01 \\
\hline Turismo Sexual & 01 \\
\hline
\end{tabular}

Fonte: Elaborado pelas autoras, 2019.

Nos artigos analisados, o storytelling foi utilizado como uma ferramenta para o

${ }^{2}$ Uma das autoras, esteve em duas publicações, mas foi filiada a três universidades diferentes. 
marketing de destinos: uma estratégia que potencializa a experiência do turista no destino (Eskilsson \& Högdahl, 2009; Pera, 2014; Howison, Higgins-Desbiolles, \& Sun, 2017; Lund, Cohen, \& Scarles, 2017; Manthiou, Kang, \& Hyun, 2017; Tung, Cheung, \& Law, 2017; Zhong, Busser, \& Baloglu, 2017; Youssef, Leicht, \& Marongiu, 2018); uma narrativa que agrupa a oferta do destino (Olsson, Therkelsen, \& Mossberg, 2016), que comunica a história do lugar (Mossberg, 2008; Smith, 2015), que divulga experiências turísticas (Argod, 2014; Akgun, Keskin, Ayar, \& Erdoğan, 2015), e que auxilia a criação de imagens do destino pelos turistas (Hsu, Dehuang, \& Woodside, 2009; Chang, Huang, \& Chen, 2013; Hsiao, Lu, Lan, 2013; Mathisen \& Prebensen, 2013).

Em específico sobre os efeitos do storytelling na criação da imagem, Mathisen e Prebensen (2013) consideram que as histórias devem ser analisadas como ferramentas promocionais devido, principalmente, às habilidades em descrever as nuances dos destinos, bem como seus valores na conjuntura da experiência turística. O storytelling é considerado uma ferramenta poderosa na propagação de valores e de significados reais para as experiências vivenciadas.

Dois estudos ainda destacam interlocutores específicos: o turista como construtores de narrativas (Chronis, 2012) e os guias de turismo como contadores de histórias (Bryon, 2012). Assim, pela perspectiva dos destinos tem-se os turistas como o personagem central em uma narrativa encenada pelas DMOs (Destination Management Organizations) em que os gestores de marketing utilizam a estrutura das histórias na criação, por exemplo, de um mundo de sonhos no qual os turistas podem ficar imersos (Mossberg, 2008). De outro modo, Pera (2014) apresenta a problemática do empoderamento dos viajantes a partir do compartilhamento de suas narrativas de viagem, pois atualmente os turistas, denominados criativos, não consomem apenas passivamente os destinos que visitam, mas tornam-se personagens ativos na produção e compartilhamento de suas próprias experiências.

\subsection{O uso do storytelling sob a perspectiva do destino turístico}

O emprego do storytelling sob a perspectiva de análise no destino turístico está sendo evidenciada por diversos autores, os quais: Olsson, Therkelsen e Mossberg (2016); Howison, Higgins-Desbiolles e Sun (2017); Lund, Cohen e Scarles (2017); Youssef, Leicht e Marongiu, (2018). Os destinos são, essencialmente, paisagens onde as histórias podem transformar espaços, de outra forma indiferentes, em destinos turísticos atraentes (Chronis, 2008), tornandoo visível e único (Hsu, Dehuang, \& Woodside, 2009).

No entanto, em vez de identificar o destino como o protagonista, os profissionais de 
marketing podem posicioná-lo como um facilitador para o visitante ser um protagonista, enfrentando e vencendo antagonistas e alcançando resultados arquétipos específicos (Woodside $\&$ Megehee, 2009). Toda grande história precisa de forças de antagonismo que os protagonistas precisam confrontar em sua busca por objetivos desejados (Mckee, 2016). Contar histórias sobre um protagonista em uma jornada pode, portanto, ser usado por DMOs para comercializar o destino, uma vez que desperta emoções e energia do público (Woodside \& Megehee, 2009), onde o sucesso de uma apresentação narrativa nos destinos turísticos depende do envolvimento dos turistas, da disposição e da capacidade de participar ativamente na experiência narrativa (Chronis, 2012; Lund, Cohen, \& Scarles, 2017).

Howison, Higgins-Desbiolles e Sun (2017) exploram a importância do storytelling e ligações da cultura Maori dentro do turismo na Nova Zelândia, através de uma variedade de percepções em que a qualidade da experiência, também, é importante ao conectar os visitantes à cultura Maori. Assim, a partir de uma pesquisa que apresentou duas etapas, uma qualitativa e uma quantitativa, concluíram que as experiências de turismo dos visitantes chineses são uma oportunidade para a promoção do destino através do storytelling.

Tendo como enfoque a construção da história de um patrimônio cultural com o seu uso para o marketing do destino, Eskilsson e Högdahl (2009) apresentam a ideia de sentido micro e macro de representação (que estão presentes na história contada). No estudo, o patrimônio cultural construído é utilizado para tornar um lugar atrativo, a partir do seu uso em projetos de marketing e de desenvolvimento do destino. De mesmo modo, para Smith (2015) o storytelling se converte em uma forma de apresentar notícias e histórias para o compartilhamento das experiências culturais vivenciadas. Assim destaca o lugar como um conceito mais abrangente, pois é uma amálgama que contempla as mais diversas características do destino, as quais: paisagem, arquitetura, história e heranças culturais, bem como estruturas sociais e de relacionamento dentro da perspectiva do turismo cultural. Em tal conjuntura, o turismo cultural se caracteriza como o encontro entre a história e o patrimônio existente no destino, pois é a partir de tais pontos que se apresentam as narrativas, a paisagem e o povo inserido em tal lugar.

Argod (2014) destaca o turismo criativo que se caracteriza como uma experiência ativa de aprendizagem que permite a co-criação da experiência dos indivíduos com os destinos turísticos. Em tal perspectiva, o storytelling é empregado tanto para apresentar os destinos para os turistas quanto para que eles compartilhem suas experiências com os potenciais consumidores. Assim, o storytelling se caracteriza como uma ferramenta de marketing para o desenvolvimento de destinos que buscam através da criatividade desenvolver produtos e serviços que conquistem os consumidores nos mais diversos níveis utilizando-se, 
principalmente, dos guias de turismo.

Do mesmo modo, Akgün, Keskin, Ayar e Erdoğan (2015) ponderam que o storytelling possui papel fundamental na construção das estratégias de marketing, pois pode ser considerado como a ferramenta mais rápida e efetiva para desenvolver novas perspectivas para as marcas. O storytelling é considerado uma arte que descreve, de modo real ou imaginário, os mais diversos eventos (incluso aqui as viagens) mediante o emprego de palavras, fotos e áudio. De fato, o storytelling pode desenvolver diferentes funções, as quais: partilhar normas e valores; desenvolver confiança e comprometimento; compartilhar e estocar conhecimentos tácitos; facilitar o aprendizado; gerar conexões emocionais; estimular a imaginação e iniciar mudanças necessárias. De tal modo, as narrativas de viagem se tornaram produtos relevantes do storytelling em que a experiência é contada e compartilhada com os leitores. Em resumo, os autores concluem que o storytelling pode ser usado como uma ferramenta de marketing para os destinos turísticos, pois trabalha como uma fonte de motivação, bem como como uma forma de desenhar a intenção de consumo e de visita dos turistas a determinada localidade.

Lund, Cohen e Scarles (2017) evidenciam a presença do storytelling nas redes sociais, em que emerge uma nova realidade, onde as marcas dos destinos turísticos são cada vez mais o produto e ao mesmo tempo, o resultado das experiências compartilhadas. Sua pesquisa sugere uma nova interpretação sobre como essas redes sociais funcionam para gerar engajamento e estimular a circulação de histórias de marca, oferecendo uma estrutura conceitual baseada nos conceitos sociológicos de narrativa, performance, performatividade e mobilidade, com o foco no turista gerando histórias, utilizando estratégias de branding, co-criando produtos e improvisando performances. E, dessa forma, fornecem uma nova abordagem para interpretar e analisar as redes sociais e marcas de mídias sociais, propondo uma teoria sobre como os destinos turísticos podem adaptar suas práticas de branding a esse novo ambiente social.

Mathisen e Prebensen (2013) inferem que o storytelling busca engajar os consumidores para que estes possam construir memórias que façam sentido frente a experiência vivenciada. Pois, a partir de tal perspectiva o destino pode alcançar o 'topo da mente' do turista, pois suas mensagens ao serem propagadas a partir do storytelling ganham força e destaque nas intenções de compra do consumidor. O estudo busca mediante o emprego de um filme promocional analisar o impacto do storytelling tanto na criação da imagem quanto na intenção de compra do consumidor descobrindo-se que tal ferramenta é útil quando se debate ambos contextos.

Assim vale destacar o trabalho de Mossberg (2008) sobre o uso de narrativas como uma ferramenta competitiva em situações de consumo turístico, ou no servicescape, ao apresentar como as organizações e as localidades podem ser conceituadas como histórias. Essas histórias 
podem ser de diferentes tipos, referir-se à marca, à história sobre a história, ao consumidor ou a um conceito desenvolvido. Assim, o storytelling pode ser utilizado para comunicar histórias em diferentes níveis, do estratégico (para elucidar o porquê de a empresa existir) ao uso no marketing (para apresentar como a organização se diferencia no mercado).

O papel do guia de turismo como contador de histórias e o uso de suas narrativas para o marketing é inserido por Bryon (2012) em seu estudo, que considera que a busca e o uso de histórias são importantes, uma vez que a interpretação tem sido mais importante que o conhecimento sobre o destino e sobre o conteúdo provido pelos guias de turismo. A partir de uma pesquisa qualitativa exploratória (foram realizadas 65 entrevistas em profundidade com guias de turismo) foram estabelecidos quatro tipos de guias de turismo: guias oficiais, guias alternativos, guias empresariais (comerciais ou de eventos) e guias relacionais (particular ou guias residentes). Apesar das diferenças, o estudo conclui que o engajamento, o entusiasmo e a paixão estão presentes em todos os tipos de guias, assim como a história do lugar fazem parte da narrativa de todos.

Smith (2015) complementa tal perspectiva ao destacar a importância da internet no contexto do storytelling, pois permite que o turista possa, juntamente com o destino, construir suas próprias narrativas disseminando, assim, novas visões a respeito dos lugares vivenciados durante as viagens. Uma perspectiva relevante é que boas histórias não devem apenas reportar, mas construir uma nova perspectiva a partir do ouvinte, pois para que o storytelling seja efetivo deve-se senti-lo e não apenas observá-lo.

Com a internet a experiência de viagem, dos mais diversos consumidores, torna-se acessível para a sociedade desenhando, assim, os comportamentos de compra dos futuros turistas. Assim, o storytelling é considerado uma poderosa ferramenta para a construção de valor no contexto dos serviços turísticos (Pera, 2014).

\subsection{O uso do storytelling sob a perspectiva do turista}

Outra vertente pode ser destacada nos estudos analisados e que desenvolve pesquisas de storytelling relacionadas ao turismo, na perspectiva do turista como produtores de histórias e narrativas de viagem, porém, evidenciando seu comportamento e experiência. O storytelling foi documentado na pesquisa de turismo comportamental destacando a conjuntura do turista em diversos momentos, sendo os principais autores: Woodside, Cruickshank, e Dehuang (2007); Woodside \& Megehee (2009); Hsu, Dehuang e Woodside (2009); Chang, Huang e Chen (2013); Hsiao, Lu e Lan (2013); Pera (2014); Smith (2015); Manthiou, Kang e Hyun (2017); Tung, Lin, Zhang e Zhao (2017); Zhong, Busser e Baloglu (2017). 
Através da narrativa, os turistas podem reviver as experiências de suas viagens. As histórias que os visitantes contam frequentemente incluem suas explicações acerca das fotografias que captam e opiniões consideradas interessante de relatar a outros (Hsu, Dehuang, \& Woodside, 2009). Chronis (2012) estuda a ativa participação dos turistas na construção de narrativas de consumo, em que atuam como visitantes analíticos e críticos e não somente como receptores passivos de informação. Concebe assim a ideia de turistas como produtores de histórias, que por sua vez são construções compartilhadas de experiências turísticas.

É com esse entendimento que Zhong, Busser e Baloglu (2017) evidenciam que o storytelling é uma ferramenta concebida como central para a experiência turística, ainda que sua relação com a experiência do turismo memorável, não tenha sido investigada empiricamente. Dentro de tal contexto, pesquisaram 400 turistas que relembraram suas mais recentes viagens de lazer e investigaram empiricamente as relações entre satisfação, comprometimento afetivo e comportamento de storytelling.

O storytelling auxilia os consumidores a encontrarem elevados níveis de satisfação, principalmente, quando compartilham suas experiências mediante a descrição de seus sentimentos, humores, sensações, percepções, interpretações e aprendizados frente ao que foi experienciado em determinada localidade e/ou empreendimento. Logo, empregar narrativas convincentes cria, dentro da perspectiva do marketing a partir do storytelling, valores que geram serviços, relacionamentos e experiências próprias. Os profissionais de marketing, em tal contexto, devem desenvolver plataformas e ferramentas que estejam aptas para que o turista compartilhe suas experiências mediante a divulgação do storytelling, ou seja, de narrativas de viagem (Pera, 2014)

O storytelling, a partir do que aborda Chang, Huang e Chen (2013), atua para desenvolver produtos que possuem significados tanto para a construção da imagem da cidade quanto para o debate a respeito da experiência vivenciada no destino visitado. Em seu estudo a respeito do turismo cultural e a importância do storytelling para o desenvolvimento de produtos que contemple as narrativas a respeito da memória e dos eventos históricos vivenciados em determinada localidade, concluíram que o emprego de produtos voltados à perspectiva cultural, se justifica devido à sua característica emocional que combina o apelo cultural com a criatividade das ideias a serem desenvolvidas.

Hsiao, Lu e Lan (2013) salientam a importância dos blogs de viagem como fonte de informação, principalmente, ao que diz respeito a propagação de informações a respeito das experiências vivenciadas pelos consumidores nos mais diversos destinos turísticos. O storytelling, em tal conjuntura, se caracteriza como uma fonte propulsora de ação para as 
atividades comerciais, principalmente, àquelas ligadas aos destinos turísticos. Os turistas utilizam-se do storytelling para propagar, prioritariamente, em seus blogs experiências vivenciadas durante o consumo do destino. Logo, tal ferramenta faz referência a maneira como o marketing pode empregar os mais diversos conteúdos para desenvolver produtos e serviços cada vez mais conectados ao consumidor e as suas experiências de vida. Utilizando-se de fotografias, layouts de blogs convidativos, design e, até mesmo áudios para desenvolver memórias afetivas nos leitores que ao buscarem um blog de viagem online querem conhecer determinado destino turístico através do olhar de outro ser humano que tenha experienciado tal situação anteriormente.

Hsu, Dehuang e Woodside (2009) analisaram as narrativas que os consumidores publicam utilizando a netnografia de marca, de forma a entender que a marca do lugar pode ser construída, entendida e promovida como um mito. Para tanto, investigaram como os visitantes interpretam locais, pessoas e situações que vivenciaram durante sua viagem e, por conseguinte como essas narrativas podem ser utilizadas para construir um destino em uma marca ícone. A partir de fotografias e da compilação das histórias publicadas pelos visitantes em blogs, foi feito um mapeamento de acordo com a relação entre um conceito com outro, podendo ser pessoas, lugares, resultados, crenças e relações positivas e negativas. Como implicações, os autores destacam que entender essas interpretações podem ser utilizadas pelos gestores para compreenderem até que ponto a estratégia de posicionamento do destino está de acordo com as imagens e as narrativas construídas pelos visitantes.

Em complementação, Martin, Woodside e Dehuang (2007) e Woodside, Cruickshank e Dehuang (2007), também, analisam as narrativas publicadas em blogs de viagens utilizando a netnografia da marca, visto que consideram que é necessário suplantar a ideia do marketing convencional de que basta um posicionamento da marca (a partir de seus atributos e benefícios) e partir para uma estratégia de marca que forneça mitos atrativos para influenciar visitantes que buscam informações on-line. O uso da netnografia foi apontado como uma oportunidade de coletar dados de forma não obstrusiva, visto que são significados atribuídos pelos visitantes quando da experiência no destino e, que uma vez postados se tornam informação pública.

Manthiou, Kang e Hyun (2017) evidenciam que sua pesquisa representou um estudo pioneiro ao incluir a recordação e o storytelling como o elo que liga duas perspectivas teóricas, analisando a experiência através destes dois constructos no turismo de cruzeiros. Com base em uma revisão de literatura, 14 hipóteses teóricas foram derivadas e um modelo de equações estruturais foi desenvolvido para serem testadas usando dados coletados de 300 passageiros de cruzeiros de luxo. Os resultados confirmaram a integração positiva dos dois aspectos teóricos 
e o estudo ao fornecer orientação aos profissionais da indústria de cruzeiros no desenvolvimento de estratégias gerenciais e de marketing.

\section{CONSIDERAÇÕES FINAIS}

O desenvolvimento e crescimento das tecnologias de informação e comunicação, as mudanças demográficas, a transformação nos mercados de turismo e a rápida mudança dos interesses dos turistas estão revolucionando profundamente o contexto do turismo nacional e internacional (Salazar, Van Den Branden, \& Bryon, 2009).

$\mathrm{Na}$ atividade turística, o equilíbrio entre oferta e demanda é um fator crucial para a inovação. Os turistas representam o lado da demanda, enquanto o lado da oferta consiste em prestadores de serviços que oferecem acomodações, alimentos, souvenirs, visitas guiadas entre outros. Para maximizar o desempenho, os turistas devem estar pelo menos satisfeitos com sua experiência, enquanto os empreendedores e prestadores de serviços devem obter um retorno satisfatório do investimento através dos produtos e serviços que fornecem (Gallouj \& Weinstein, 1997). Além disso, o principal tipo de "produto" no turismo tem a ver com os sentimentos das pessoas. Estamos nos encaminhando para uma sociedade em que as pessoas estão dispostas a pagar mais por uma emoção do que por um determinado ativo tecnológico (Jensen, 2001). Essa economia da experiência é um condutor claro por trás de formas inovadoras de turismo, porque a demanda por histórias é maior do que a que pode ser encontrada atualmente (todos os marcos contêm histórias específicas que podem atrair turistas). Os empreendedores turísticos de sucesso há muito entendem a importância capital de passar da entrega de serviços para as experiências de preparação (que vendem) (Salazar, Van Den Branden, \& Bryon, 2009).

Com efeito e no contexto em que o marketing tem sido liderado pela pesquisa e uso intensivo da tecnologia de informação e comunicação (Buhalis, 2000), o storytelling se tornou uma ferramenta importante para o marketing turístico, para a interpretação do turismo e para a experiência turística (Howison, Higgins-Desbiolles, \& Sun, 2017). O storytelling enquanto técnica de pesquisa pode encadear cenas, proporciona um sentido envolvente para captar a atenção das pessoas, formando um quadro memorável, ou seja, uma ferramenta de comunicação estruturada em uma sequência de acontecimentos que apelam aos sentidos e emoções (Xavier, 2015).

Como tópico de pesquisa, o storytelling está sendo amplamente discutido na literatura (Sidali, Spiller, \& Schulze, 2011) o que traz à tona a complexidade do conceito e o uso de uma definição universalmente aceita. Outra consideração são os conceitos que emergem do uso do 
storytelling nos estudos em turismo. Hsu, Dehuang e Woodside (2009) ao analisarem as narrativas que os consumidores publicam consideraram a ideia de emic interpretation, ou seja, a interpretação relativa à histórias contadas em primeira pessoa, em que o protagonista conta como, quando e porque os eventos ocorreram e que, em última instância pode ser caracterizada como o que as pessoas constroem e trazem consigo das viagens e dos destinos que visitam. Por sua vez, Martin, Woodside e Dehuang (2007) destacaram a ideia de etic interpretation, ou seja, interpretar as interpretações dos visitantes, suas experiências no destino a partir da narrativa que apresentam. Desse modo, assim como destacado por Chronis (2012) compreendemos que o conhecimento do lugar pode ser medido pelo quanto sabemos sobre suas histórias.

Em tal conjuntura, o storytelling pode e deve ser trabalhado como uma ferramenta que enriquece a comunicação entre os destinos turísticos e seus consumidores (turistas), pois é a partir deste que se criam conexões positivas entre ambos os protagonistas. Assim, estudos que envolvam a compreensão do storytelling e o seu impacto na experiência turística são relevantes para a construção de um corpus de pesquisa cada vez mais atual no cenário acadêmico.

\section{REFERÊNCIAS}

Adaval, R., \& Wyer, R. S., Jr. (1998). The role of narratives in consumer information processing. Journal of Consumer Psychology, 7(3), 207-245.

Akgun, A. E., Keskin, H., Ayar, H., \& Erdoğan, E. (2015). The influence of storytelling approach in travel writings on readers' empathy and travel intentions. Procedia Social and Behavioral Sciences, 207, 577-586.

Albach, V. M. (2015) A difusão da pesquisa em geografia do turismo na Ibero-América. Tese de Doutorado, Universidade Federal do Paraná, Curitiba, PR, Brasil.

Alcantud-Díaz, M., Vayá, A. R., \& Gregori-Signes, C. (2014). 'Share your experience'. Digital storytelling in English for tourism. Ibérica, 27, 185-204.

Alves, F. G., Costa, H. S., \& Perinotto, A. R. C. (2017). Instagram como ferramenta para fidelização de clientes: Fotografia, Redes Sociais e Turismo. Marketing \& Tourism Review, 2 (2), 1-21.

Argod, P. (2014). Arts visuels et médiation d'un tourisme créatif: de l'expérience du voyage, de la pratique artistique et des "creatifs culturels". Mondes du Tourisme, 10, 47-61.

Barrett, H.C. (2006a). Researching and evaluating digital storytelling as a deep learning tool. In C.M. Crawford et al. (Eds.), 647-654.

Barrett, H. C. (2006b). Digital stories in ePortfolios: Multiple purposes and tools.

Recuperado em 19, maio, 2018 de http://electronicportfolios.org/digistory/purposesmac.html

Boje, D. M. (1995). Stories of the storytelling organization: a postmodern analysis of Disney 
as “Tamara- Land”. Academy of Management Journal, 38(4), 997-1035.

Broome, M. E. (2000). Integrative literature reviews for the development of concepts. In B. L. Rodgers, \& K. A. Knafl (Orgs.), Concept development in nursing: foundations, techniques, and applications, 231- 250. Philadelphia, PA: W. B. Saunders.

Bryon, J. (2012). Tour Guides as Storytellers: from selling to sharing. Scandinavian Journal of Hospitality and Tourism, 12(1), 27-43.

Buhalis, D. (2000). Marketing: the competitive destination of the future. Tourism Management, 21, 97-116.

Chang, T-Y., Huang, K-L., \& Chen, K-H. (2013). Culture-driven products: Creating cultural engagement with historical heritage. Applied Mechanisms and Materials, 311, 366-371.

Chronis, A. (2008). Co-constructing the narrative experience: staging and consuming the American Civil War at Gettysburg. Journal of Marketing Management, 24(1-2), 05-27.

Chronis, A. (2012). Tourists as story-builders: Narrative construction at a Heritage Museum. Journal of Travel \& Tourism Marketing, 29(5), 444-459.

Clarke M., \& Horton R. (2001). Bringing it all together: Lancet-Cochrane collaborate on systematic reviews. Lancet, 357(9270), 1728.

Delgadillo, Y., \& Escalas, J. E. (2004). Narrative word-of mouth communication: Exploring memory and attitude effects of consumer storytelling. Advances in Consumer Research, 31,186-192.

Drew, M., \& Woodside, A. G. (2011). Storytelling research on international visitors Interpreting own experiences in Tokyo. Qualitative Market Research: An International Journal, 14(1), 27-54.

Eskilsson, L., \& Högdahl, E. (2009). Cultural Heritage across borders? - Framing and challenging the Snapphane Story in Southern Sweden. Scandinavian Journal of Hospitality and Tourism, 9(1), 65-80.

Fog, K., Budtz, C., Munch, P., Blanchette, S. (2010). Storytelling: Branding in Practice. Berlin: Springer.

Fog, K., Budtz, C., \& Yakaboylu, B. (2005). Storytelling: Branding in practice. Berlin: Springer.

Gabriel, Y. (2000). Storytelling in Organizations. Facts, Fictions, and Fantasies. Oxford: Oxford University Press.

Gallouj, F., \& Weinstein, O. (1997). Innovation in services. Research Policy, 26(4-5), 537556.

Garrett, M. T. (1996). “Two People": An American Indian narrative of bicultural identity. Journal of American Indian Education, 36(1), 1-21.

Guedes, V. L. S., \& Borschiver, S. (2005). Bibliometria: uma ferramenta estatística para a gestão da informação e do conhecimento, em sistemas de informação, de comunicação e de avaliação científica e tecnológica. Anais do CINFORM - Encontro Nacional de Ciência da Informação, Salvador, 06. 
Howison, S., Higgins-Desbiolles, F., \& Sun, Z. (2017). Storytelling in tourism: Chinese visitors and Māori hosts in New Zealand. Anatolia, 28(3), 327-337.

Hsiao, K-L., Lu, H-P., \& Lan, W-C. (2013). The influence of the components of storytelling blogs on readers' travel intentions. Internet Research, 23(2), 160-182.

Hsu, S-Y., Dehuang, N., \& Woodside, A. G. (2009). Storytelling research of consumers' selfreports of urban tourism. Journal of Business Research, 62, 1223-1254.

Hull, G.A., \& Nelson, M. E. (2009). Literacy, media, and morality: Making the case for an aesthetic turn. In M. Prinsloo \& M. Baynham (Eds.) The Future of Literacy Studies, 199-227. Houndmills: Palgrave Macmillan.

Jensen, R. (1999). Dream society: how the coming shift from information to imagination will transform your business. Journal of Consumer Policy, 20, 289-323.

Jensen, R. (2001). The Dream Society: How the Coming Shift from Information to Imagination Will Transform Your Business. New York: McGraw-Hill.

Kim M. H., \& Jeong H. G. (2010). The study on development of local dishes in Chungcheongnam-do through storytelling: centered on King Muryeong and King Tamra and Mongyudowonbangbap. Journal of The Korean Society of Food Culture, 25(3), 213-232.

Lambert, J. (2006). Digital Storytelling: Capturing Lives, Creating Community. (2nd Ed.). Berkeley, CA: Digital Diner Press.

Lee, Y-S., \& Shin, W-J. (2015). Marketing tradition-bound products through storytelling: a case study of a Japanese sake brewery. Service Business, 9(2), 281-295.

Lopes, A. L. M., \& Fracolli, L. A. (2008). Revisão Sistemática de Literatura e Metassíntese Qualitativa: Considerações sobre sua aplicação na pesquisa em enfermagem. Texto \& Contexto em Enfermagem, 17(4), 771-778.

Lund, N. F, Cohen, S. A., \& Scarles, C. (2017). The power of social media storytelling in destination branding. Journal of Destination Marketing \& Management.

Macedo, M., Botelho, L. L. R., \& Duarte, M. A. T. (2010). Revisão Bibliométrica sobre a produção científica em aprendizagem gerencial. Revista Eletrônica Gestão e Sociedade, 4(8), $1-21$.

Manthiou, A., Kang, J., \& Hyun, S. S. (2017). An integration of cognitive appraisal theory and script theory in the luxury cruise sector: the bridging role of recollection and storytelling. Journal of Travel and Tourism Marketing, 1-18.

Martin, D., Woodside, N., \& Dehuang, N. (2007). Etic Interpreting of naïve subjective personal introspections of tourism behavior: Analyzing visitors' stories about experiencing Mumbai, Seoul, Singapore, and Tokyo. International Journal of Culture, Tourism and Hospitality Research, 1(1), 14-44.

Mathisen, L., \& Prebensen, N. K. (2013). Dramatizing an event through a promotional film: testing image effects. Journal of Travel \& Tourism Marketing, 30(7), 672-689.

McCabe, S., \& Foster, C. (2006). The Role and Function of Narrative in Tourist Interaction. Journal of Tourism \& Cultural Change, 4(3), 194-215. 
Mckee, R. (2016). Dialogue: The art of verbal action for page, stage, and screen. New York, NY: Twelve.

Montoro-Sanchez, A. (2009). Competitive dynamics and business models in service business: a promising research subject. Service Business, 3(4), 311-318.

Mossberg, L. (2008). Extraordinary experiences through storytelling. Scandinavian Journal of Hospitality and Tourism, 8(3), 195-210.

Moscardi, E., Pinto, M., Gomes, E., \& Nakatani, M. (2017). O uso das revisões bibliométrica, sistemática e integrativa de literatura para compreender o conceito de informação turística. Revista Turismo e Desenvolvimento, 27/28, 1821-1830.

Nakatani, M. S. M., Gomes, E. L., \& Nunes, M. P. (2017). Diferentes Olhares da Comunicação no Turismo: entendendo três localidades paranaenses como destino e produto turístico. Revista Turismo em Análise, 28(3), 474-491.

National Storytelling Network. (2011). What is Story-telling? Recuperado em 20, maio, 2018, de http://www.storynet.org/resources/whatisstory-telling.html

Nielsen, J. K. (2017). Museum Communication and storytelling: articulating understandings within the museum structure. Museum Management and Curatorship, 32(5), 440-455.

O’Gorman, K. D., \& Gillespie, C. (2010). The mythological power of hospitality leaders? A hermeneutical investigation of their reliance on storytelling. International Journal of Contemporary Hospitality Management, 22(5), 659-680.

Olsson, A. K., Therkelsen, A., \& Mossberg, L. (2016). Making an effort for free - volunteers' roles in destination-based storytelling. Current Issue in Tourism, 19(7), 659-679.

Patterson, A., \& Brown, S. (2005). No Tale No Sale: A Novel Approach to Marketing Communication. The Marketing Review, 5(4), 315-328.

Pera, R. (2014). Empowering the new traveller: storytelling as a co-creative behaviour in tourism. Current Issues in Tourism, 20(4), 331-338.

Powell, T. B., Weems, W., \& Owle, F. (2007). Native American digital storytelling: Situating the Cherokee oral tradition within American literary history. Literature Compass, 4(1), 1-23.

Price, M., Kallam, M., \& Love, J. (2009). The learning styles of Native American students and implications for classroom practice. Retrieved May, 19, 2018, from www.se.edu/nas/files/2013/03/NAS-2009-Proceedings-M-Price.pdf

Robin, B. (2006). The educational uses of digital storytelling. In C.M. Crawford et al. (Eds.), 709-716.

Robin, B. (2008a). The effective uses of digital storytelling as a teaching and learning tool. In J. Flood, S. Heath \& D. Lapp (Eds.). Handbook of Research on Teaching Literacy through the Communicative and Visual Arts, 429-440. New York: Lawrence Erlbaum.

Robin, B. (2008b). Digital storytelling: A powerful technology tool for the 21 st century classroom. Theory into Practice, 47, 220-228.

Russel, C. L. (2005). An overview of the integrative research review. Progress in Transplantation, 15(1), 8-13. 
Salazar, N. B., Van Den Branden, E., \& Bryon, J. (2009). Cultural tourism storytelling in 'Flanders': The story behind the stories. Haverle: Steunpunt Toerisme.

Sidali, K., Spiller, A., \& Schulze, B. (2011). Food, agriculture and tourism: Linking local gastronomy and rural tourism: Interdisciplinary perspectives. Berlin: Springer Science and Business Media.

Smith, S. (2015). A sense of place: place, culture and tourism. Tourism Recreation Research, 40(2), 220-233.

Souza, C. D. (2013). A organização do conhecimento: estudo bibliométrico na base de dados ISI web of knowledge. Biblios, 108(51).

Tung, V.W.S., Cheung, C., \& Law, R. (2017) Does the Listener Matter? The Effects of capitalization on storytellers' evaluations of travel memories. Journal of Travel Research.

Tung, V. W. S., Lin, P., Zhang, H. Q., \& Zhao, A. (2017). A framework of memory management and tourism experiences. Journal of Travel \& Tourism Marketing, 34(7), 853866.

Xavier, A. (2015) Storytelling: Histórias que deixam marcas. São Paulo: Best Business.

Wainberg, J. A. (2003). Turismo e Comunicação: a indústria da diferença. São Paulo: Contexto.

Whittemore, R., \& Knafl, K. (2005). The integrative review: updated methodology. Journal of Advanced Nursing, 52, 546-553.

Woodside, A. G., Cruickshank, B. F., \& Dehuang, N. (2007). Stories visitors tell about Italian cities as destination icons. Tourism Management, 28, 162-174.

Woodside, A. G., \& Megehee, C. M. (2009). Travel Storytelling Theory and Practice. Anatolia, 20(1), 86-99.

Youssef, K. B., Leicht, T., \& Marongiu, L. (2018). Storytelling in the context of destination marketing: an analysis of conceptualizations and impact measurement. Journal of Strategic Marketing, 1-18.

Zhong, Y. Y., Busser, J., \& Baloglu, S. (2017). A model of memorable tourism experience: the effects on satisfaction, affective commitment, and storytelling. Tourism Analysis, 22, 201217.

\section{FORMATO PARA CITAÇÃO DESTE ARTIGO}

MANOSSO, F. C., RUIZ, T. C. D., \& NAKATANI, M. S. M. (2020). A aplicação do Storytelling nas pesquisas em Turismo: Uma Revisão Bibliométrica, Sistemática e Integrativa da Literatura. Revista de Turismo Contemporâneo, 8(2), 337-358. https://doi.org/10.21680/2357$\underline{8211.2020 v 8 n 2 I D 16465}$ 